\title{
On Randomness in Quantum Mechanics
}

\author{
Alberto C. de la Torre* \\ Departamento de Física, \\ Universidad Nacional de Mar del Plata \\ Funes 3350, 7600 Mar del Plata, Argentina \\ CONICET
}

\begin{abstract}
The quantum mechanical probability densities are compared with the probability densities treated by the theory of random variables. The relevance of their difference for the interpretation of quantum mechanics is commented.
\end{abstract}

\section{INTRODUCTION}

Probabilities were introduced in quantum mechanics by Max Born[1] when he proposed an interpretation for the wave function $\Psi(x)$ of a particle as a probability amplitude. Thereby $|\Psi(x)|^{2}$ is a probability density, that is, the probability assigned to the event corresponding to the location of the particle in the interval $[x, x+d x]$. Today, 80 years later, we don't know the nature of this probability, that is, we don't know whether this probability has an ontological or a gnoseological character: we don't know whether the particle is located somewhere but we can not know where it is, and the best that we can do is to give a probability for it, or on the contrary, the very location of the particle is diffuse by nature. Although the Bell-Kochen-Specker theorem [2, 3] favours an ontological interpretation, as can be easily seen in a recent illustration[4] of the theorem concerning position and momentum observables, the opposite view, assuming context dependent definite values for the observables, is not excluded. It soon became clear however that these probabilities do not behave as such for mutually exclusive events and the rule "thou shall not add the probabilities but their amplitudes instead" was adopted although never understood. A beautiful illustration of the necessity of this rule is given by R. Feynman [5] in the analysis of the two slits experiment.

Quantum mechanical "probabilities" are not added; however, probabilities are studied by a rigourous mathematical theory of random variables that tell us that, indeed, they can be added. Instead of requiring a change in the mathematical theory we should perhaps accept that the quantum mechanical densities are not probabilities. In this work we will analyse the quantum mechanical densities and compare them with the probability densities defined in the theory of random variables. We will see that, in general, the quantum mechanical densities do not behave as the probability densities of random variables and we will comment on the consequences of this for the interpretation of quantum mechanics.

*Electronic address: delatorre@mdp.edu.ar 


\section{RANDOM VARIABLES}

For completeness, we will recall some concepts of the theory of random variables. A random variable $A$ is a mathematical entity that may be assigned numerical values $a$ with some probability. We can use the symbol $A \Rightarrow a$ to denote the event that the random variable is assigned a value in the interval $[a, a+d a]$. We define the Probability Density Function $\varrho(a)$ as the probability of realization or appearance of the event $A \Rightarrow a$. Of course, theses densities are nonnegative and normalized.

Probabilities play an important role in classical physics and are omnipresent in quantum mechanics so, in some cases, it is interesting to consider the observables of a system as random variables with their corresponding probability density functions. For instance, we may study the possibility of assuming that the position of a particle $X$ and its momentum $P$ are random variables with their associated probability densities $\varrho(x)$ and $\pi(p)$ that give the probability of location of the particle and of having some value of momentum. Since position and momentum are two independent concepts, in the sense that a particle at a given position can have any value of momentum, and some value of momentum can be realized at any position, we can assume that position and momentum are described by independent random variables. We will later mention the possibility that they are not independent.

Given a random variable $A$ with density $\varrho(a)$, a function $H(A)$ of the random variable $A$, will have a density $\eta(h)$ given by

$$
\eta(h)=\int d a \varrho(a) \delta(h-H(a))
$$

This result is intuitive: the probability for some value $h$ is the probability of the event $A \Rightarrow a$ times the probability that $h=H(a)$, which is a sharp Dirac distribution, added for al possible values of $a$. Similarly, it is easy to understand that if $A$ and $B$ are independent random variables and $\varrho(a)$ and $\pi(b)$ are the corresponding probability densities associated with the events $A \Rightarrow a$ and $B \Rightarrow b$, then the probability density $\sigma(c)$ assigned to the random variable $C=F(A, B)$ is given by

$$
\sigma(c)=\int d a \int d b \varrho(a) \pi(b) \delta(c-F(a, b)) .
$$

This result can not be avoided. If by any reason, the event $C \Rightarrow c$ doesn't have the given probability density, it does not means that the theory is wrong but it suggests that, perhaps, $\varrho(a)$ and $\pi(b)$ are not the probability densities of the random variables $A$ and $B$.

\section{QUANTUM RANDOMNESS}

We will prove the following theorem. Assume two observable of a quantum system represented by the operators $A$ and $B$ in a Hilbert space. Let $\varrho(a)$ and $\pi(b)$ be the distributions of the eigenvalues of the operators as predicted by quantum mechanics for the system in a state $\Psi$, that is, $\varrho(a)=\left\langle\Psi, P_{a} \Psi\right\rangle$ where $P_{a}$ is the projector in the invariant subspace of the eigenvalue $a$ (in the case of nondegeneracy of the eigenvalue $a$ with eigenvector $\varphi_{a}$, we have $P_{a}=\varphi_{a}\left\langle\varphi_{a}, \bullet\right\rangle$ and we get the more familiar expression $\left.\varrho(a)=\left|\left\langle\varphi_{a}, \Psi\right\rangle\right|^{2}\right)$ and similarly, $\pi(b)=\left\langle\Psi, P_{b} \Psi\right\rangle$ $\left(\pi(b)=\left|\left\langle\phi_{b}, \Psi\right\rangle\right|^{2}\right.$ if $b$ is nondegenerate with eigenvector $\left.\phi_{b}\right)$. Let $C=F(A, B)$ be an observable built as a function of the observables $A$ and $B$ whose operator has the eigenvalues $c$ and the corresponding projector $P_{c}$ (eigenvector $\xi_{c}$ if 
nondegenerate). Then, considering the quantum mechanical distribution $\sigma_{Q M}(c)$ and the probability density provided by the theory of random variables $\sigma_{R V}(c)$ given by

$$
\begin{aligned}
\sigma_{Q M}(c) & =\left\langle\Psi, P_{c} \Psi\right\rangle \\
\sigma_{R V}(c) & =\int d a \int d b \varrho(a) \pi(b) \delta(c-F(a, b))
\end{aligned}
$$

we can prove the following:

1. if $[A, B] \neq 0$ then in general it is $\sigma_{Q M}(c) \neq \sigma_{R V}(c)$;

2. if $[A, B]=0$ then we have two cases:

(a) if they commute because they are related by a function like $B=G(A)$, then $\sigma_{Q M}(c)=\sigma_{R V}(c)$;

(b) if they commute because they are independent observables related to different degrees of freedom, then we have again two cases:

i. if the state is factorizable in the product Hilbert space corresponding to the different degrees of freedom, then $\sigma_{Q M}(c)=\sigma_{R V}(c)$;

ii. if the state is not factorizable (entangled) in the product Hilbert space corresponding to the different degrees of freedom, then $\sigma_{Q M}(c) \neq \sigma_{R V}(c)$.

Summarizing we have

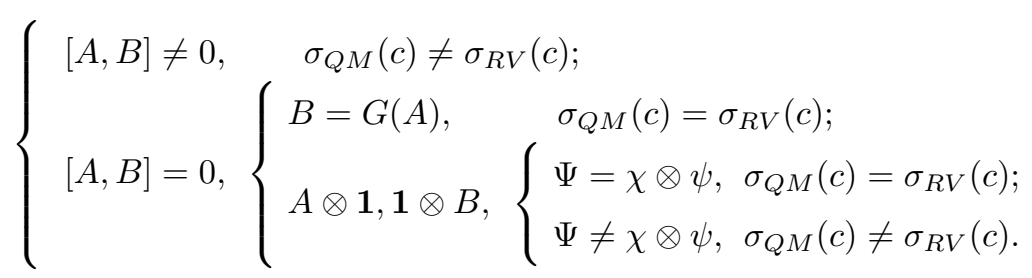

That is, in general the quantum mechanical distribution and the random variables densities are different and only in some cases (commuting operators and factorizable state) they can be equal.

In order to compare them, we can write Eqs.(3) and (4) in a way to emphasize their difference (assuming nondegenerate eigenvalues $a, b$ and $c$ ) as

$$
\begin{aligned}
\sigma_{Q M}(c) & =\int d a \int d b\left\langle\varphi_{a}, \Psi\right\rangle\left\langle\Psi, \phi_{b}\right\rangle\left\langle\phi_{b}, \quad \xi_{c}\left\langle\xi_{c}, \bullet\right\rangle \quad \varphi_{a}\right\rangle \\
\sigma_{R V}(c) & =\int d a \int d b\left\langle\varphi_{a}, \Psi\right\rangle\left\langle\Psi, \phi_{b}\right\rangle\left\langle\phi_{b}, \quad \delta(c-F(a, b)) \Psi\langle\Psi, \bullet\rangle \quad \varphi_{a}\right\rangle,
\end{aligned}
$$

The QM case involves the projector in the state $\xi_{c}$ whereas in the RV case it involves the projector in the state $\Psi$ and a constraint $\delta(c-F(a, b))$. Of course this formal difference only suggests that $\sigma_{Q M} \neq \sigma_{R V}$ but it does not prove it because the different operators are inside an integral. We will later prove anyway that they are indeed different. A nice comparisons of the two distributions (3) and (4) is obtained when we calculate the moments of the distributions. For the QM case we have

$$
E_{Q M}^{(n)}=\int d c c^{n} \sigma_{Q M}(c)=\left\langle\Psi, \int d c c^{n} P_{c} \Psi\right\rangle=\left\langle\Psi, C^{n} \Psi\right\rangle=\left\langle\Psi, F^{n}(A, B) \Psi\right\rangle,
$$


and in the RV case we have

$$
E_{R V}^{(n)}=\int d c c^{n} \sigma_{R V}(c)=\int d c c^{n} \int d a \int d b \varrho(a) \pi(b) \delta(c-F(a, b))=\int d a \int d b F^{n}(a, b) \varrho(a) \pi(b) .
$$

Inserting the completeness relation in Eq.(8) and replacing the quantum mechanic expression for the densities $\varrho$ and $\pi$ we get

$$
\begin{aligned}
E_{Q M}^{(n)} & =\int d a \int d b\left\langle\Psi, P_{a} F^{n}(A, B) P_{b} \Psi\right\rangle, \\
E_{R V}^{(n)} & =\int d a \int d b\left\langle\Psi, P_{a} F^{n}(a, b) P_{\Psi} P_{b} \Psi\right\rangle .
\end{aligned}
$$

Notice that the QM case involves the operator $F^{n}(A, B)$ and the RV case the number $F^{n}(a, b)$ multiplied by the projector in the state $\Psi$. A nicer comparison is obtained in the particular case where $[A, B]=0$. In this case we can write $F^{n}(A, B)$ as a power series with the operator $B$ always to the right and $A$ to the left and using the relations $B P_{b}=b P_{b}$ and $P_{a} A=P_{a} a$ we have

$$
[A, B]=0 \rightarrow\left\{\begin{array}{l}
E_{Q M}^{(n)}=\int d a \int d b F^{n}(a, b)\left\langle\Psi, \quad P_{a} P_{b} \Psi\right\rangle=\int d a \int d b F^{n}(a, b) \quad \operatorname{Tr}\left\{P_{a} P_{b} P_{\Psi}\right\} \\
E_{R V}^{(n)}=\int d a \int d b F^{n}(a, b)\left\langle\Psi, P_{a} P_{\Psi} P_{b} \Psi\right\rangle=\int d a \int d b F^{n}(a, b) \operatorname{Tr}\left\{P_{a} P_{\Psi} P_{b} P_{\Psi}\right\}
\end{array}\right.
$$

where we have used the trace expression for the expectation values. Clearly the moments, and therefore the distributions, are different in general although for some states they may be equal (for instance when $P_{b}$ and $P_{\Psi}$ commute).

Considering some examples it is clear that for noncommuting observables, like position and momentum, it must be $\sigma_{Q M} \neq \sigma_{R V}$. Take for instance $A=X^{2}, B=P^{2}$ and $C=X^{2}+P^{2}$ where $\varrho(a)$ and $\pi(b)$ are continuous, and therefore $\sigma_{R V}(c)$ is also continuous, whereas $\sigma_{Q M}(c)$ is not continuous because it corresponds to the energy distribution of an harmonic oscillator.

Another case of physical interest with noncommuting observables is when $A=X$, the position of a free particle at $t=0, B=\frac{t}{m} P$, its velocity multiplied by the time $t$, and $C=A+B=X+P \frac{t}{m}$ corresponds to the position of the particle at time $t$. The quantum mechanical prediction for the distribution of position at time $t$ clearly differs from the random variable distribution. To see this we can, for instance, calculate the width of both distributions $\Delta^{2}=E^{(2)}-\left(E^{(1)}\right)^{2}$ using Eqs.(8,9), or directly from Eqs. $(3,4)$, and we obtain

$$
\begin{aligned}
\Delta_{Q M}^{2} & =\Delta_{x}^{2}+\frac{t^{2}}{m^{2}} \Delta_{p}^{2}+\frac{t}{m}(\langle\Psi,(X P+P X) \Psi\rangle-2\langle\Psi, X \Psi\rangle\langle\Psi, P \Psi\rangle), \\
\Delta_{R V}^{2} & =\Delta_{x}^{2}+\frac{t^{2}}{m^{2}} \Delta_{p}^{2}
\end{aligned}
$$

where $\Delta_{x}$ and $\Delta_{p}$ are the width of the probability densities of position and momentum $\varrho(x)$ and $\pi(p)$. The conceptual relevance of this difference will be discussed later. Notice that even if the observables $A$ and $B$ would commute, we would still get different results in some states where the corresponding correlation term in Eq.(13) does not vanish.

Let us consider now the case of $[A, B]=0$ because $B=G(A)$. In this case, if we know the density $\varrho(a)$ for the random variable $A$ then we calculate the density of $B$ using Eq.(1) as

$$
\pi(b)=\int d a \varrho(a) \delta(b-G(a))
$$

This result of the theory of random variables is also obtained in quantum mechanics where $\pi(b)=\left\langle\Psi, P_{b} \Psi\right\rangle$. If $[A, B]=0$, the operators $\mathrm{A}$ and $B$ share eigenvectors, that is, they have the same projectors. However the function 
$G(A)$ can introduce more degeneracy because there may be different values $a \neq a^{\prime}$ with $G(a)=G\left(a^{\prime}\right)$ corresponding to the same eigenvalue $b$ (think for instance that for $X^{2}$ we have $P_{x^{2}}=P_{x}+P_{-x}$ ). Therefore the projector $P_{b}$ is obtained adding all projectors $P_{a}$ where $a$ and $b$ satisfy the condition $b=G(a)$. That is,

$$
P_{b}=\int d a P_{a} \delta(b-G(a))
$$

Now, taking the expectation value $\left\langle\Psi, P_{b} \Psi\right\rangle$ we immediately obtain the relation in Eq. (15). In this case, the observable $C=F(A, B)$ does not really depends on $A$ and $B$ but is a function of just one observable $C=F(A, G(A))$ and repeating the same argument it is clear that the theory of random variables and quantum mechanics predict the same density: $\left\langle\Psi, P_{c} \Psi\right\rangle=\int d a \varrho(a) \delta(c-F(a, G(a)))$.

We can now analyse the case where the observables $A$ and $B$ commute because they correspond to different degrees of freedom. These operators act in different Hilbert spaces $\mathcal{H}_{1}$ and $\mathcal{H}_{2}$ and the system is described by a state in the space $\mathcal{H}=\mathcal{H}_{1} \otimes \mathcal{H}_{2}$. Given a state $\Psi$, there is a basis $\left\{\chi_{k}\right\}$ in $\mathcal{H}_{1}$ and a basis $\left\{\zeta_{k}\right\}$ in $\mathcal{H}_{2}$ that allow the bi-orthogonal decomposition of the state

$$
\Psi=\sum_{k=1}^{N} \alpha_{k} \chi_{k} \otimes \zeta_{k}
$$

As is well known, if $N=1$ the state is factorizable and if $N \geq 2$ the state is entangled or nonfactorizable. The operators and their corresponding projectors are extended in the product space as $A \otimes \mathbf{1}, P_{a} \otimes \mathbf{1}, \mathbf{1} \otimes B, \mathbf{1} \otimes P_{b}$, and the densities associated with the observables are given by quantum mechanics as $\varrho(a)=\left\langle\Psi, P_{a} \otimes \mathbf{1} \Psi\right\rangle$ and $\pi(b)=\left\langle\Psi, \mathbf{1} \otimes P_{b} \Psi\right\rangle$. The operator $C=F(A, B)$ will have projectors $P_{c}$ associated with the eigenvalues $c$ that project in a subspace of $\mathcal{H}_{1} \otimes \mathcal{H}_{2}$. We will prove that

$$
P_{c}=\int d a \int d b \delta(c-F(a, b)) P_{a} \otimes P_{b}
$$

In order to prove it, let us consider the function $F(A, B)$ expanded as a formal power series

$$
C=F(A, B)=\sum_{n, m} c_{n, m} A^{n} \otimes B^{m}
$$

Now, from the spectral decomposition of $A=\int d a a P_{a}$ and using the orthogonality and idempotent property of the projectors $P_{a} P_{a^{\prime}}=\delta\left(a-a^{\prime}\right) P_{a}$ we easily find that $A^{n}=\int d a a^{n} P_{a}$ and similarly $B^{m}=\int d b b^{m} P_{b}$. Replacing above and reconstructing the function we get

$$
C=F(A, B)=\int d a \int d b F(a, b) P_{a} \otimes P_{b} .
$$

Now we write the function $F(a, b)$ as an integral over $c$ with a Dirac distribution

$$
C=F(A, B)=\int d a \int d b \int d c c \delta(c-F(a, b)) P_{a} \otimes P_{b}=\int d c c \int d a \int d b \delta(c-F(a, b)) P_{a} \otimes P_{b} .
$$

But this is precisely the spectral decomposition of the operator $C$ and therefore the double integral over $a$ and $b$ is the projector $P_{c}$ as given in Eq.(18). We obtain the quantum mechanical distribution of the eigenvalues of the operator $C$ by taking the expectation value in Eq. (18)

$$
\sigma_{Q M}(c)=\left\langle\Psi, P_{c} \Psi\right\rangle=\int d a \int d b \delta(c-F(a, b))\left\langle\Psi, P_{a} \otimes P_{b} \Psi\right\rangle
$$


In this expression we consider now the two cases for the state, factorizable or entangled. In the first case the state is given by $\Psi=\chi \otimes \zeta$ and inserting above we have

$$
\sigma_{Q M}(c)=\int d a \int d b \delta(c-F(a, b))\left\langle\chi, P_{a} \chi\right\rangle\left\langle\zeta, P_{b} \zeta\right\rangle
$$

but $\left\langle\chi, P_{a} \chi\right\rangle=\left\langle\Psi, P_{a} \otimes \mathbf{1} \Psi\right\rangle=\varrho(a)$ and $\left\langle\zeta, P_{b} \zeta\right\rangle=\pi(b)$ and therefore

$$
\sigma_{Q M}(c)=\int d a \int d b \delta(c-F(a, b)) \varrho(a) \pi(b),
$$

which is the density predicted by the theory of random variables; that is, $\sigma_{Q M}(c)=\sigma_{R V}(c)$. In the non-factorizable case we have

$$
\sigma_{Q M}(c)=\int d a \int d b \delta(c-F(a, b)) \sum_{k, r} \alpha_{k}^{*} \alpha_{r}\left\langle\chi_{k}, P_{a} \chi_{r}\right\rangle\left\langle\zeta_{k}, P_{b} \zeta_{r}\right\rangle
$$

which contains all non-diagonal terms. Notice that here $\varrho(a)=\sum_{k}\left|\alpha_{k}\right|^{2}\left\langle\chi_{k}, P_{a} \chi_{k}\right\rangle$ and $\pi(b)=\sum_{k}\left|\alpha_{k}\right|^{2}\left\langle\zeta_{k}, P_{b} \zeta_{k}\right\rangle$ and then

$$
\sigma_{R V}(c)=\int d a \int d b \delta(c-F(a, b)) \sum_{k, r}\left|\alpha_{k}\right|^{2}\left|\alpha_{r}\right|^{2}\left\langle\chi_{k}, P_{a} \chi_{k}\right\rangle\left\langle\zeta_{r}, P_{b} \zeta_{r}\right\rangle
$$

therefore in general we have $\sigma_{Q M}(c) \neq \sigma_{R V}(c)$.

\section{CONCLUSION}

The general conclusion that we can draw from this study is that quantum mechanics is not a random process in space. If it where, then the randomness in quantum mechanics should be described by the theory of random variables and we have seen that this is not the case.

In particular, we saw in the last section that if we know the probability density for the position of a particle $\varrho(x)$ at one time, and we know the probability density of its velocity (or momentum) $\pi(p)$, then, according to quantum mechanics, the probability density for the position at a later time is not calculated by sampling one position and adding a displacement sampled from the velocity distribution, as would be calculated with the theory of random variables. Notice that this result contradicts the gnoseological interpretation of the probability for position and velocity of the particle. Indeed, if the particle has a definite position, unknown to us, with its value distributed according to $\varrho(x)$, and the particle has a definite velocity, unknown to us and distritbuted according to $\pi(p)$, then the position at a later time must given by the rule " $x_{0}+v t$ " where $x_{0}$ and $v$ are taken from their distributions. In other words, the location at time $t$ must be given according to the theory of random variables; but this is contrary to the quantum mechanical result! This can only mean that $X$ and $P$ are not random variables and $\varrho(x)$ and $\pi(p)$ are not densities of probabilities although they are, in principle, measured as if they were, that is, by counting the frequency of realization of the events $X \Rightarrow x$ and $P \Rightarrow p$. To call "probability" to something that is not a probability, that is, something that does not complies with the rules of probabilities, is perhaps a misuse of language and it could be convenient to denote the quantum mechanical distributions with another name. It is unfortunate that the term "probability" is irreversibly installed in quantum mechanics because, strictly speaking, they are not probabilities and the misnomer introduces confusion, not only in the teaching, but also in search of an interpretation of quantum mechanics. A more 
appropriated name for $\varrho(x)$ and $\pi(p)$ could be for instance the existential weight of the events $X \Rightarrow x$ and $P \Rightarrow p$ in the system in a particular state. Although these existential weights are measured in an experiment in the same way that one measures a probability, these quantities are not probabilities and do not necessarily obey all the rules of probabilities dictated by the theory of random variables. Probabilities propagate according to the theory of random variables but the existential weights are calculated by the rules of quantum mechanics. Accordingly we can think about the position of a particle as a diffuse observable without a putative value. Every possible value, every event $X \Rightarrow x$, has a propensity to appear in an observation given by the existential weight, but this does not means that the event has that value with some probability and that the measurement of the observable exhibits a pre-existent value. This idea has been synthetically expressed by A. Peres saying that "unperformed experiments have no result" [6].

The results presented in this work suggest that we can not consider $\varrho(x)$ and $\pi(p)$ to be the probability densities of two independent random variables associated with the position and momentum of a particle. We should consider now the possibility that position and momentum are not independent and are related by some unknown connection that is responsible for the quantum mechanical correlations. We could then recover the theory of random variables for quantum mechanics if we could show that $\varrho(x)$ and $\pi(p)$ are the marginal distributions of a joint probability density $W(x, p)$. There are many possibilities to achieve this, the most famous is the Wigner phase space distribution, but it was shown [7] that the most general function producing the same expectation values as quantum mechanics is not nonnegative everywhere and therefore it can not be considered as a genuine joint probability density.

\section{ACKNOWLEDGEMENTS}

This work received partial support from "Consejo Nacional de Investigaciones Científicas y Técnicas" (CONICET), Argentina.

[1] M. Born, "Zur Quantenmechanik de Stossvorgänge" Zeitschrift für Physik 37 863-867 (1926).

[2] J. S. Bell, "On the problem of hidden variables in quantum theory," Rev. Mod. Phys. 38, 447-452 (1966).

[3] S. Kochen and E. P. Specker, "The problem of hidden variables in quantum mechanics," J. Math. Mech. 17, 59-88 (1967).

[4] A. C. de la Torre, "Observables have no value: a no-go theorem for position and momentum observables" ArXiv: quant-ph/8798798, Found. of Phys. xx, xxx-xxx (2007). DOI 10.1007/s10701-007-9148-2

[5] R. P. Feynman, R. B. Leighton and M. Sands The Feynman Lectures on Physics, Quantum Mechanics, Volume III. Chapter 1. Addison-Wesley, Reading (1965).

[6] A. Peres, "Unperformed experiments have no result", Am. J. Phys 46, 745-747 (1978).

[7] L. Cohen, "Generalized phase-space distribution functions", Journal of Mathematical Physics 7, 781-786 (1966). 